\title{
Autopercepção de saúde bucal por idosos marajoaras
}

\author{
Self-perception of buccal health by marajoaras elderly people
}

\author{
Danielle Tupinambá Emmi ${ }^{1}$ \\ Karem Menezes Gomes ${ }^{2}$ \\ Regina Fátima Feio Barroso ${ }^{1}$ \\ Marizelli Viana de Aragão Araújo ${ }^{1}$ \\ Helder Henrique Costa Pinheiro ${ }^{1}$
}

\section{RESUMO}

A autopercepção em saúde refere-se à experiência subjetiva sobre o seu bem-estar funcional, social e psicológico. Assim, o objetivo deste estudo foi investigar a autopercepção quanto à saúde bucal de uma população de idosos não institucionalizada residente no Arquipélago do Marajó, região norte do Brasil. Configura-se um estudo descritivo transversal com uma amostra do tipo aleatória simples $(\mathrm{n}=50)$. A autopercepção foi avaliada por meio de questionário validado pelo SB Brasil 2010. Avaliou-se também o uso e necessidade de prótese, mediante exame clínico bucal. A associação entre a autopercepção, fatores clínicos de uso e necessidade de prótese e fatores socioeconômicos foi verificada por meio do teste do Quiquadrado, com $\alpha=0,05$. De acordo com os resultados obtidos, $84 \%$ dos idosos já procurou atendimento odontológico, sendo $51,2 \%$ no serviço particular, principalmente para fazer extração dentária $(55,8 \%)$. Observou-se que, dos idosos que não usavam prótese, $75 \%$ tinham apenas até 4 anos de aproveitamento de estudos $(p=0,031)$ e que 59,4\% tinham renda familiar total entre $R \$ 501$ a $R \$ 1.500$ reais $(p=0,234)$. Apesar de não utilizarem a prótese, $62 \%$ consideraram que necessitavam utilizar o dispositivo $(\mathrm{p}=0,003)$, visto que $56 \%$ sentiam dificuldades para falar $(\mathrm{p}=0,015)$. Contudo, $54 \%$ não sentiam vergonha em falar ou sorrir $(\mathrm{p}=0,869)$. Percebeu-se que a necessidade de prótese dentária é clinicamente superior à percebida pelos idosos marajoaras participantes da pesquisa, e dentro dessas condições, é configurado como consequência de um contexto multifatorial que abrange variáveis socioeconômicas, geográficas e culturais.

Palavras-chave: Idoso. Autopercepção. Prótese dentária. Saúde bucal.

\begin{abstract}
Self-perception in health refers to subjective experience about their functional, social and psychological well-being. Thus, the objective of this study was to investigate the self-perception regarding oral health of a non-institutionalized elderly population living in the Marajó Archipelago, northern region of Brazil. A descriptive cross-sectional study was performed with a simple random sample $(n=50)$. Self-perception was evaluated by means of a questionnaire validated by SB Brazil 2010. The use and necessity of a prosthesis was also evaluated through oral clinical examination. The association between self-perception, clinical factors of use and need for prosthesis and socioeconomic factors was verified using the chi-square test, with $\alpha=0.05$. According to the results obtained, $84 \%$ of the elderly already sought dental care, and $51.2 \%$ performed it in the private service, mainly to make dental extraction $(55.8 \%), 75 \%$ had only 4 years of study use $(\mathrm{p}=0.031)$ and $59.4 \%$ had a total family income of between $\mathrm{R} \$ 501$ and $\mathrm{R} \$ 1,500(\mathrm{p}=0.234)$. Although they did not use the prosthesis, $62 \%$ considered that they needed to use the device $(\mathrm{p}=0.003)$, since $56 \%$ had difficulty speaking $(\mathrm{p}=0.015), 54 \%$ did not feel ashamed to speak or smile $(\mathrm{p}=0.869)$. It was noticed that the need for dental prosthesis is clinically superior to that perceived by the marajoara elderly participants of the research and within these conditions is configured as a consequence of a multifactorial context that encompasses socioeconomic, geographic and cultural variables.
\end{abstract}

Keyword: Elderly. Self-perception. Dental prosthesis. Oral health.

\footnotetext{
${ }^{1}$ Professor (a) Doutor (a), Faculdade de Odontologia, Universidade Federal do Pará

${ }^{2}$ Cirurgiã-dentista
} 


\section{INTRODUÇÃO}

Segundo dados do Instituto Brasileiro de Geografia e Estatística (IBGE), baseado na última Pesquisa Nacional de Amostra de Domicílios (PNAD), o percentual de pessoas com 60 anos ou mais na população brasileira passou de $12,8 \%$ para $14,4 \%$, entre 2012 e $2016^{1}$. Isso reflete um crescimento de $16,0 \%$ na população dessa faixa etária, passando de 25,5 milhões para quase 30 milhões. Essa mudança por qual passa a sociedade brasileira, é reflexo de uma combinação de fatores relacionados à melhora nas condições básicas de vida, avanço técnico-científico e até o acesso aos serviços de saúde. Neste contexto, convém destacar que o aumento de idosos na população requer uma atenção diferenciada de diversos setores da sociedade, especialmente dos profissionais da saúde ${ }^{2}$.

Durante muitas décadas a atenção à saúde bucal foi caracterizada e voltada ao atendimento juvenil escolar, enfatizando a cárie e a doença periodontal nesta faixa etária. Os demais grupos populacionais eram assistidos apenas em casos de emergência odontológica, tornando a atenção em saúde bucal um modelo de exclusão, composto de práticas que visavam aspectos curativos e remediadores, com análises insuficientes das reais necessidades odontológicas dos idosos brasileiros ${ }^{3}$.

Dos problemas bucais existentes no paciente idoso, o edentulismo é um dos mais frequentes ${ }^{4}$. A gravidade da perda dentária nesses indivíduos expressa o acúmulo das doenças bucais mais prevalentes (cárie e doença periodontal) e sua maior sequela, revelando a ineficiência e ineficácia dos programas voltados para os idosos durante muitas décadas ${ }^{5}$.

A perda dentária atribui dentro da variedade de domínio físico, social e psicológico, fatores como a capacidade e restrição alimentar, descontentamento dos indivíduos em relação ao aproveitamento das refeições no meio social, bem como a ocorrência de dor e desconforto mastigatório ${ }^{6}$. Damasceno et $\mathrm{al}^{7}$ configuram a estética dental como um aspecto para autoconfiança e autoestima a serem considerados quando se analisa o indivíduo em sua autopercepção e interação social. Assim, evidencia-se que a perda dentária está associada à redução da qualidade de vida ${ }^{8}$.
Considerando o enfoque na saúde bucal da população idosa pode-se perceber que há uma interação entre as condições bucais desses indivíduos e sua qualidade de vida, onde essa interação não está somente relacionada às consequências físicas, mas também às questões sociais e psicológicas?

Tradicionalmente, as pesquisas relacionadas ao diagnóstico das condições de saúde das populações têm sido realizadas apenas por indicadores numéricos, e, a determinação das necessidades, apenas do ponto de vista normativo, ignorando aspectos sociocomportamentais que deveriam ser considerados ${ }^{10}$.

Sendo assim, é importante analisar a interpretação que uma pessoa faz de seu estado de saúde e as suas experiências no contexto de sua vida diária ${ }^{11}$. Martins et al. ${ }^{12}$ afirmaram que a autopercepção da saúde é um componente amplamente considerável no que diz respeito à saúde bucal, pois será responsável em acrescentar normas sociais e culturais, considerando a importância dada pelo indivíduo na necessidade do tratamento odontológico.

Apesar da importância da autopercepção da saúde bucal, há pouca utilização dessa medida como fator preponderante no que diz respeito à avaliação do indivíduo ${ }^{13}$. Em detrimento a este fato faz-se necessária, então, a inclusão da percepção da saúde em estudos a serem realizados. Estudos de autopercepção em saúde permitem que a população tenha conhecimento de suas debilidades em relação à saúde, e assim possa auxiliar na formulação de exigências públicas direcionadas às suas necessidades ${ }^{10}$.

O Arquipélago do Marajó é considerado o maior arquipélago fluvial e marítimo do mundo. $\mathrm{O}$ conjunto das ilhas marajoaras contrasta sua diversidade e extensão com agravantes no que diz respeito à situação socioeconômica, com municípios com os menores Índices de Desenvolvimento Humano (IDH), em relação aos demais municípios brasileiros ${ }^{14,15}$. Dentre os municípios que compõem o Arquipélago do Marajó, foram escolhidos cinco para serem estudados nesta pesquisa, os quais não foram enfoque de nenhum levantamento com relação à saúde bucal até o presente momento, mesmo com o baixo padrão de qualidade de vida da sua população. A escolha dos municípios deveu- 
se a estes apresentarem estrutura mínima de Odontologia e Índice de Desenvolvimento Humano (IDH) semelhante aos demais municípios da Ilha, de acordo com dados do IBGE contidos no Plano de Desenvolvimento Territorial Sustentável do Arquipélago do Marajó ${ }^{14,15}$.

Assim, o presente estudo teve por objetivo avaliar a autopercepção em saúde bucal de idosos não institucionalizados residentes no Arquipélago do Marajó, região Norte do Brasil e analisar a associação entre as condições socioeconômicas e psicossociais desses idosos, com dados clínicos de uso e necessidade de prótese.

\section{Metodologia}

Este estudo se constituiu de uma pesquisa seccional de base populacional, realizado durante o período de 2011 a 2013, nos municípios de Breves, Curralinho, São Sebastião da Boa Vista, Soure e Salvaterra, localizados no Arquipélago do Marajó, Estado do Pará.

Considerando que o grupo populacional de 65 a 74 anos passa a ser de fundamental importância para a verificação das necessidades de tratamento e do controle dos efeitos gerais dos cuidados odontológicos prestados a essa faixa de idade, foram selecionados 50 idosos entre 65 a 74 anos, não institucionalizados, residentes nos municípios envolvidos na pesquisa. O tamanho da amostra foi calculado utilizando como referência paramétrica a prevalência da cárie dentária e margem de erro de 10\% (intervalo de confiança de 95\%).

As quatro equipes de campo formadas, cada uma com um examinador e um anotador, foram treinadas em oficina de trabalho e submetidas a processo de calibração, sendo obtido ótimo coeficiente de concordância entre examinadores.

Todos os exames foram realizados mediante a aceitação do indivíduo e prévia obtenção do Termo de Consentimento Livre e Esclarecido (TCLE) aprovado pelo Comitê de Ética em Pesquisa do Instituto de Ciências da Saúde da Universidade Federal do Pará (CEP - ICS/UFPA - Parecer n ${ }^{\circ} 85 / 2009$ ).
Os exames bucais foram realizados em espaços reservados nas unidades de saúde ou em espaços domiciliares, utilizando-se espátula de madeira, espelho clínico e gaze, sob luz natural. Os dados foram anotados em ficha clínica adaptada da Pesquisa Nacional de Saúde Bucal SB Brasil 2010.

Para avaliação da autopercepção em saúde bucal dos idosos utilizou-se formulário validado pela Pesquisa Nacional de Saúde Bucal - SB Brasil 2010.

Os dados foram apurados no Programa Computacional Microsoft Office Excel 2010. A estatística descritiva e a associação entre as variáveis foram desenvolvidas no programa Statistical Package for the Social Scienses (SPSS), com nível de significância de 5\% e intervalo de confiança de $95 \%$. Foi utilizado o teste do Qui-quadrado para medir a associação entre o uso e necessidade de prótese e fatores socioeconômicos e de autopercepção em saúde bucal dos idosos.

\section{Resultados}

A Tabela 1 sintetiza a descrição quanto ao acesso e utilização do tratamento odontológico pelos idosos participantes da pesquisa. Nos achados quanto ao comparecimento a um consultório odontológico, $84 \%$ dos entrevistados foram ao dentista pelo menos uma vez, sendo que a maioria (60\%) realizou a última visita ao dentista há aproximadamente 3 anos, principalmente no serviço particular $(51,2 \%)$, para realizar algum tipo de tratamento dentário, onde a extração foi o motivo principal $(55,8 \%)$.

Considerando a importância do fator socioeconômico na saúde bucal, foi feita a associação entre os fatores socioeconômicos e o uso clínico da prótese dentária, o que é visualizado na Tabela 2 . Observa-se que a maioria dos entrevistados relatou renda familiar no mês anterior à entrevista, entre $\mathrm{R} \$ 501$ a $\mathrm{R} \$ 1.500$ reais totalizando $52 \%$ da população amostral, em que 19 indivíduos não faziam uso de nenhuma prótese $(59,4 \%)$ e 7 indivíduos usavam prótese em pelo menos uma das arcadas $(38,9 \%)$. 
Entre os idosos, a faixa de escolaridade que aparece com o maior percentual é a que corresponde a até quatro anos de estudo, com $62 \%$, considerando que deste percentual, $75 \%$ dos indivíduos não usavam prótese em nenhuma arcada e 38,9\% usavam prótese em pelo menos uma das arcadas, o que mostra significância estatística $(\mathrm{p}=0,031)$ (Tabela 2).

Na Tabela 3 relaciona-se a autopercepção dos idosos quanto a saúde bucal e fatores psicossociais. Observouse que $72 \%$ dos entrevistados considerou, no momento da pesquisa, que necessitava de atendimento odontológico. Entretanto, 53,1\% dos indivíduos que não utilizavam prótese consideraram que não necessitavam utilizá-la, enquanto $88,9 \%$ dos que já utilizavam a prótese em pelo menos uma das arcadas, consideraramse insatisfeitos com o dispositivo, mencionando que necessitavam trocar a prótese atualmente usada $(\mathrm{p}=0,003)$.

Constata-se também na Tabela 3, a autopercepção da dificuldade em falar relacionada à causa dentária em 68,8\% daqueles que não faziam o uso da prótese, mostrando uma associação estatística significante $(\mathrm{p}=0,015)$. Considerando fatores psicológicos como relevantes na procura ao atendimento odontológico, $46 \%$ dos idosos sentiam vergonha de falar ou sorrir, principalmente aqueles que não utilizavam prótese dentária (46,9\%). Dentre os entrevistados, 32\% estabeleceu uma relação negativa entre os seus dentes e as atividades de trabalho/escola $(\mathrm{p}=0,036)$.

Com relação à necessidade clínica do uso de prótese dentária e a relação com o fator socioeconômico, a Tabela 4 mostra que $88,9 \%$ dos idosos demonstraram necessidade do uso de Prótese Parcial Removível (PPR) e 72,7\% indivíduos necessitavam de Prótese Total (PT) em pelo menos uma das arcadas. Observou-se que 63\% dos indivíduos que necessitavam de PPR e 40,9\% dos que necessitavam de PT possuíam renda familiar total, no mês anterior à entrevista, entre $R \$ 501$ e $R \$ 1.500$ reais, entretanto estes dados não mostraram significância estatística $(\mathrm{p}=0,304)$.

A necessidade de tratamento dentário no momento da pesquisa foi evidenciada por $81,5 \%$ dos indivíduos que necessitavam de PPR e 63,6\% dos que necessitavam de PT, entretanto, $36 \%$ dos idosos ainda referiram não necessitar do uso de prótese, mesmo sendo esta necessidade constatada clinicamente (Tabela 5).

\section{DisCUSSÃo}

O aumento da população idosa vem sendo configurado no mundo todo, e no Brasil não foge à regra. Este fenômeno ocorre de forma acelerada, dentro de um quadro de assistência à saúde ainda pouco preparado para suprir as condições necessárias no que diz respeito à oferta de serviços para melhora da qualidade de vida $\mathrm{e}$ manutenção da saúde desse grupo etário. Neste aspecto, percebe-se a importância de determinantes sociais, econômicos, organizacionais, culturais e ambientais ${ }^{16}$, visto que estes podem influenciar a procura e o acesso aos serviços de saúde.

A avaliação do uso e necessidade de prótese ajuda a entender o agravo "edentulismo", servindo, ao mesmo tempo, para estimar a gravidade do problema, pela análise conjunta dos dados de uso e necessidade, assim como, para subsidiar ações de planejamento a partir da análise das necessidades ${ }^{17}$. Se por um lado o emprego do índice apropriado permite a identificação objetiva da necessidade normativa do uso de prótese dentária, por outro, a adoção destes índices não leva em conta a efetiva demanda (necessidade subjetiva) do indivíduo pelo uso destas próteses. Essa necessidade subjetiva é influenciada não apenas pela ausência de elementos dentários, mas principalmente por fatores como idade do paciente, conforto, custo do tratamento, preferências individuais, diferenças culturais e acesso aos serviços de saúde ${ }^{18,19}$.

Neste estudo, dentre os idosos caracterizados com necessidade de prótese, seja PPR ou PT, 12 entrevistados (24\%) acreditavam não necessitar de tratamento dentário e, mesmo sendo constatado clinicamente a necessidade de reabilitação protética, 18 (36\%) ainda referiram não necessitar do uso (Tabela 5). Esse tipo de situação, diagnosticada nos exames clínicos, é assintomática e desconhecida pelos pacientes, que muitas vezes só consideram que necessitam de atenção em saúde, diante das manifestações agudas das doenças bucais ${ }^{12}$. Além disso, pode-se considerar que 
indicadores objetivos medem doença, enquanto os subjetivos avaliam experiências humanas e saúde ${ }^{20}$. Corroborando com esses resultados, Henriques et al. ${ }^{11}$ afirmam que as análises realizadas por meio da autopercepção fornecem informações diferentes das obtidas por meio da avaliação clínica realizada pelo cirurgião-dentista e, o fato dos idosos não terem um julgamento apropriado do estado de sua saúde bucal, aliado à não percepção das necessidades se constitui em barreira de acesso ao serviço odontológico, configurando uma das principais razões para essas pessoas não procurarem o atendimento ${ }^{21}$.

A partir disso, verifica-se a grande importância da autopercepção em saúde bucal, pois o conhecimento do paciente acerca de sua saúde é o primeiro passo na elaboração de um programa que inclua ações educativas, voltadas para o autodiagnóstico e autocuidado, além de ações preventivas e curativas ${ }^{22}$.

É importante ressaltar que alguns aspectos que influenciam o acesso e a utilização dos serviços de saúde podem estar profundamente interligados, como a escolaridade e a renda. No entanto, a baixa escolaridade esteve associada com o menor uso de serviços de saúde ${ }^{3}$. No presente estudo, o baixo nível de escolaridade dos entrevistados, não mostrou relação estatística com a necessidade de prótese ( $\mathrm{p}=0,125)$, apesar de se constatar $o$ alto percentual de idosos com baixa escolaridade que necessitavam de reabilitação protética, seja de PPR $(74,1 \%)$, seja de PT (50\%) (Tabela 4).

Contrastando com essa situação, as chances de procurar serviços de saúde aumentam à medida que os indivíduos ganham anos de idade e não acumulam anos de estudo, ou seja, pessoas idosas com baixa escolaridade, apesar de usarem menos os serviços de saúde, têm maior probabilidade para necessitar desses serviços $^{23}$, pois tem menos conhecimento sobre práticas preventivas em saúde. Essa afirmativa está de acordo com os achados deste estudo, o que é observado na Tabela 1 em que, $60 \%$ da população entrevistada estava há 3 ou mais anos sem consultar o dentista.

Em relação à renda familiar, o maior percentual dos indivíduos entrevistados encontrava-se no intervalo entre $R \$ 501$ a $R \$ 1.500$ reais, sendo também neste intervalo o maior percentual de idosos $(52 \%)$ que necessitavam de prótese (Tabela 4). Junqueira et al. ${ }^{24}$ afirmam que as pessoas e áreas de pior nível socioeconômico apresentam piores condições de saúde bucal.

Nos resultados obtidos pôde-se constatar que $84 \%$ dos idosos entrevistados já foram consultados por um dentista, porém apenas $22,5 \%$ dessas consultas ocorreram há menos de um ano (Tabela 1). Apesar dos avanços do Sistema Único de Saúde (SUS), principalmente com a implantação da Estratégia Saúde da Família (ESF), o acesso à atenção odontológica necessita ser ampliado para grupos populacionais que têm como porta de entrada apenas os planos de saúde ou consultórios particulares, opções economicamente determinadas e socialmente excludentes ${ }^{3}$. O presente estudo mostrou que 51,2\% das consultas odontológicas realizadas pelos idosos aconteceram no serviço particular (Tabela 1). Estudo com relação ao uso do serviço odontológico pelos idosos mostrou um maior uso de prótese entre os usuários do serviço particular, quando comparados aos usuários do serviço público ${ }^{19}$.

O reduzido número de programas de saúde bucal voltados para idosos, o baixo índice de profissionais e com pouco preparo para o atendimento desses indivíduos em nosso país, ressaltam a necessidade de profundos estudos e adequação técnica para maior atenção a essa população ${ }^{25}$.

A partir de suas características sociais, econômicas e geográficas os municípios do Arquipélago do Marajó parecem estar inseridos no contexto de exclusão e sofrem as consequências da má distribuição, locação e da carência no suprimento necessário dos serviços públicos de saúde.

O motivo das consultas odontológicas também se configura um fator de importância para a análise direcionada à saúde bucal. Constatou-se que o motivo principal da última consulta realizada foi $55,8 \%$ para realização de extração dentária (Tabela 1). Até hoje a população idosa é a que apresenta o maior número de necessidades odontológicas acumuladas ${ }^{26}$, revelando o que, historicamente, caracterizou a prestação da atenção odontológica, com ações de baixa complexidade e na sua maioria curativas e mutiladoras ${ }^{27}$. 
Concordando com elevado percentual de necessidade de prótese obtido nessa pesquisa, o edentulismo configura-se a situação específica mais viável para a região dos municípios do Marajó, devido à falta de recursos especializados para sanar a dor de dente e de Centros de Especialidades Odontológicas. Alguns autores agregam valores sociais ao edentulismo, além da organização dos serviços de saúde bucal ao longo da história como a oferta da assistência cirúrgicorestauradora, e confirmam que em situações por problemas econômicos, os dentes eram extraídos ${ }^{12,28}$. Por muito tempo a única forma de controle da dor de dente foi remediada de forma irreversível, tornando o edentulismo um processo comum ao envelhecimento.

Os resultados obtidos neste estudo mostram que a população idosa marajoara estudada sofre consequências em relação à assistência em saúde bucal, causada pela instabilidade multifatorial encontrada em seus municípios no que rege os aspectos socioeconômicos e organizacionais. A situação de grande necessidade acumulada reflete o histórico descaso desse segmento populacional na atenção à saúde bucal em nosso país e coloca um grande desafio ao Sistema Único de Saúde (SUS), considerando o seu papel na redução das desigualdades e de provimento de acesso universal ao cuidado integral ${ }^{29}$.

A falta de acesso ao serviço público odontológico e a não utilização de próteses, mostrou um quadro de necessidades que são percebidas pelos entrevistados no que tange ao tratamento odontológico (Tabela 5), como sentir dificuldade para falar por conta da falta de elementos dentários. Porém, em detrimento a essas necessidades, não houve correlação, em maioria, na autopercepção, que implica a situação dentária como um fator de comprometimento pessoal e social e na execução de tarefas como trabalho/estudo. Isso evidencia em grande parte, a aceitação do edentulismo como evento natural, superestimando suas condições de saúde bucal. Nota-se então que a falta de percepção da necessidade de atendimento odontológico aliado à cultura de que indivíduos edêntulos, não necessitam de acompanhamento odontológico, contribuem para o número reduzido de idosos frequentadores dos serviços de saúde bucal, refletindo a falta de orientação e de cuidados com a sua saúde bucal ${ }^{25}$.

\section{Conclusão}

O fator renda familiar obteve estreita relação com o uso e necessidade de prótese, enquanto o tempo de aproveitamento escolar, a acessibilidade e a utilização dos serviços odontológicos configuram associação positiva entre esses aspectos e o uso e necessidade de prótese.

Percebeu-se que a necessidade de prótese dentária é clinicamente superior à percebida pelos idosos marajoaras participantes da pesquisa e dentro dessas condições é configurado como consequência de um contexto multifatorial que abrange variáveis socioeconômicas, geográficas e culturais.

\section{REFERÊNCIAS}

1. Instituto Brasileiro de Geografia e Estatística. Síntese de indicadores sociais: uma análise das condições de vida da população brasileira. IBGE, Coordenação de População e Indicadores Sociais. Rio de Janeiro: IBGE, 2016. 146 p.

2. Fazito LT, Perim JV, Di Ninno CQMS. Comparação das queixas alimentares de idosos com ou sem prótese dentária. Rev CEFAC. 2004; 6(2):143-50.

3. Moreira RS, Nico LS, Tomita NE, Ruiz T. A saúde bucal do idoso brasileiro: revisão sistemática sobre o quadro epidemiológico e acesso aos serviços de saúde bucal. Cad Saúde Pública. 2005; 21(6):1665-75.

4. Rosendo RA, Sousa JNL, Abrantes JGS, Cavalcante ABP, Ferreira AKTF. Saúde bucal e impacto na qualidade de vida em idosos. RSC online. 2017; 6(1):89-102.

5. Rabelo MAB, Emmi DT, Herkrath J, Meira GF, Góes PSA, Vettore MV. Need of Dental Prosthesis in Older People and the Offer of Specialized Care in the Unified Health System, Brazil. Pesq Bras Odontoped Clin Integr. 2018, 18(1):1-12.

6. Gerritsen A, Allen F, Wittter D, Bronkhorst E, Creugers N. Tooth loss and oral-health related quality of life: a systematic review and meta-analysis. Health Qual Life Outcomes. 2010; 8(126):1-11.

7. Damasceno LMCM, Marassi CS, Ramos MEB, Souza IPR. Alterações no comportamento infantil decorrente da perda de dentes anteriores: relato de caso. Rev Bras Odontol. 2002; 59(3):193-6. 
8. Mallmann F, Toassi R, Abegg C. Perfil epidemiológico do uso e necessidade de prótese dentária em indivíduos de 5074 anos de idade, residentes em três 'Distritos Sanitários' de Porto Alegre, Estado do Rio Grande do Sul, Brasil, em 2008. Epidemiol Serv Saúde. 2012; 21(1):79-88.

9. Silva DD, Sousa MLR, Wada RS. Autopercepção e condições de saúde bucal em uma população de idosos. Cad Saúde Pública. 2005; 21(4):1251-9.

10. Reis SCGB, Marcelo VC. Saúde bucal na velhice: percepção dos idosos, Goiânia, 2005. Ciênc Saúde Coletiva 2006; 11(1):191-9.

11. Henriques C, Telarolli-Júnior R, Loffredo LCM, Montandon AAB, Campos JADB. Autopercepção das condições de saúde bucal de idosos do município de Araraquara - SP. Cienc Odontol Bras. 2007; 10(3):67-73.

12. Martins AMEBL, Barreto SM, Silveira MF, Santa-Rosa TTA, Pereira RD. Autopercepção da saúde bucal entre idosos brasileiros. Rev Saúde Pública. 2010; 44(5): 91222.

13. Matos DL, Lima-Costa MF. Autoavaliação da saúde bucal entre adultos e idosos residentes na Região Sudeste: resultados do Projeto SB-Brasil, 2003. Cad Saúde Pública. 2006; 22(8):1699-707.

14. Brasil. Plano de Desenvolvimento Territorial Sustentável do Arquipélago do Marajó. Grupo Executivo Interministerial. Brasília, 2007. 296p. Disponível em: http://www.mi.gov.br/c/document_library/get file?uuid= 9408a880-6ec0-4be0-9cb7-feb01c4a6256\& groupId=249 15 [Acesso em: 2017 jun 22]

15. BRASIL. Programa das Nações Unidas para o Desenvolvimento - PNUD: índice de desenvolvimento humano municipal Brasileiro: atlas do desenvolvimento humano no Brasil. Brasília, DF: PNUD, 2016.

16. Mello AL, Moysés SJ. Melhores práticas em sistemas locais de saúde: sob foco a saúde bucal do idoso. Physis. 2010; 20(3):785-809.

17. Roncalli AG, Silva NN, Nascimento AC, Freitas CHSM, Casoti E, Peres KG, et al. Aspectos metodológicos do Projeto SBBrasil 2010 de interesse para inquéritos nacionais de saúde. Cad Saúde Pública. 2012: 28 (Supl 1):40-57.

18. Narby B, Kronstrom M, Soderfeldt B, Palmqvist S. Prosthodontics and the patient: what is oral rehabilitation need? Conceptual analysis of need and demand for prosthodontic treatment. Part 1: a conceptual analysis. Int J Prosthodont 2005; 18(1):75-9.

19. Azevedo JS, Azevedo MS, Oliveira LJC, Correa MB, Demarco FF. Uso e necessidade de prótese dentária em idosos brasileiros segundo a Pesquisa Nacional de Saúde Bucal (SBBrasil 2010): prevalências e fatores associados. Cad. Saúde Pública 2017; 33(8):e00054016

20. Locker D, Slade G. Association between clinical and subjective indicators of oral health status in an older adult population. Gerodontology. 1994; 11(2):108-14.
21. Lester V, Ashley F, Gibbons D. The relationship between socio-dental indices of handicap, felt need for dental treatment and dental state in a group of frail and functionally dependent older adults. Community Dent Oral Epidemiol. 1998; 26:155-9.

22. Silva SRC, Fernandes RC. Autopercepção das condições de saúde bucal por idosos. Rev Saúde Pública. 2001; 35(4):349-55.

23. Neri M, Soares W. Desigualdade social e saúde no Brasil. Cad Saúde Pública. 2002; 18 (Suppl.): 77-87.

24. Junqueira SR, Frias AC, Zilbovicius C. Saúde Bucal Coletiva: quadros social, epidemiológico e político. In: Rode SM, Nunes SG. Atualização clínica em Odontologia. São Paulo: Artes Médicas, 2004. p.591-604.

25. Nunes CSR, Silva MP, Barcessat ARP. Acesso aos serviços de saúde bucal de adultos e idosos. Estação Científica 2017; 7(3): 9-18.

26. Fonseca FA, Jones KM, Mendes DC, dos Santos Neto PE, Ferreira RC, Pordeus IA, et al. The oral health of seniors in Brazil: addressing the consequences of a historic lack of public health dentistry in an unequal society. Gerodontology 2015; 32(1):18-27.

27. Pucca GA, Gabriel M, de Araújo ME, de Almeida FC. Ten years of a National Oral Health Policy in Brazil: innovation, boldness, and numerous challenges. J Dent Res 2015; 94(10):1333-7.

28. Vargas AMD, Paixão HH. Perda dentária e seu significado na qualidade de vida de adultos usuários de serviço público de saúde bucal do Centro de Saúde Boa Vista, em Belo Horizonte. Ciênc Saúde Coletiva. 2005; 10(4):101524.

29. Pinto RS, Matos DL, Loyola-Filho AI. Características associadas ao uso de serviços odontológicos públicos pela população adulta brasileira. Ciênc Saúde Coletiva. 2012; 17(2):531-44. 
RDAPO: Revista Digital da Academia Paraense de Odontologia

Belém-PA, v.2, n.1, jan./jun. 2018 


\section{TABELAS}

Tabela 1. Autopercepção de idosos quanto ao acesso e utilização do serviço odontológico. Marajó, Pará, 2011-2013.

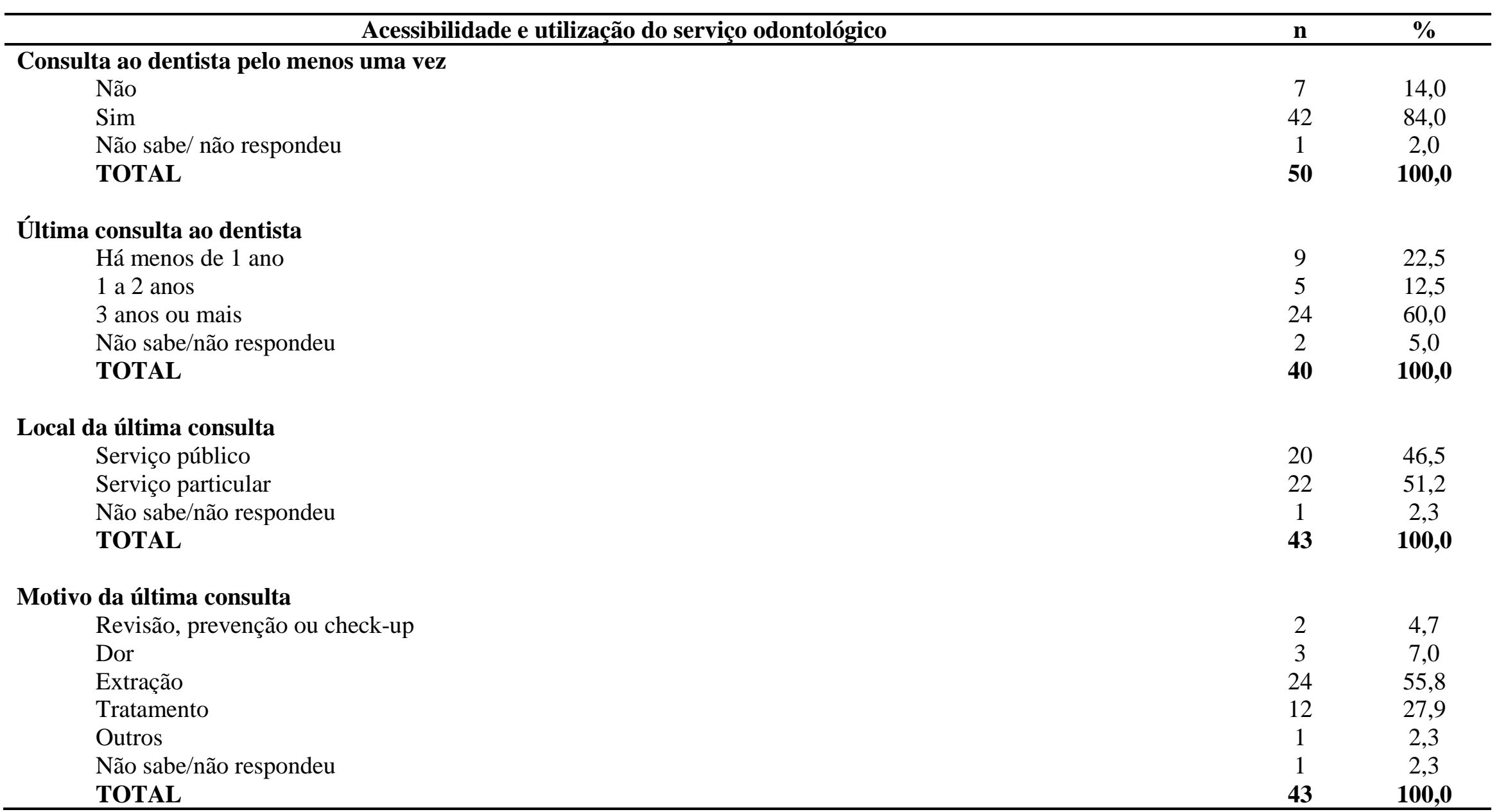


Tabela 2. Relação entre o uso clínico de prótese dentária e fatores socioeconômicos dos idosos. Marajó, Pará, 2011-2013.

\begin{tabular}{|c|c|c|c|c|c|c|c|}
\hline & & \multicolumn{6}{|c|}{ Uso de Prótese Dentária } \\
\hline & & \multicolumn{2}{|c|}{$\begin{array}{l}\text { Não usa em } \\
\text { nenhuma das } \\
\text { arcadas }\end{array}$} & \multicolumn{2}{|c|}{$\begin{array}{c}\text { Usa em pelo } \\
\text { menos uma das } \\
\text { arcadas }\end{array}$} & \multicolumn{2}{|c|}{ Total } \\
\hline & & (n) & $(\%)$ & (n) & $(\%)$ & (n) & $(\%)$ \\
\hline \multirow{8}{*}{$\begin{array}{c}\text { Renda familiar total } \\
\text { em reais do mês } \\
\text { anterior } \\
(p=0,234)^{*}\end{array}$} & Até 250 & 1 & 3,1 & 1 & 5,6 & 2 & 4,0 \\
\hline & De 251 a 500 & 7 & 21,9 & 2 & 11,1 & 9 & 18,0 \\
\hline & De 501 a 1.500 & 19 & 59,4 & 7 & 38,9 & 26 & 52,0 \\
\hline & De 1.501 a 2.500 & 1 & 3,1 & 2 & 11,1 & 3 & 6,0 \\
\hline & De 2.501 a 4.500 & 0 & 0 & 0 & 0 & 0 & 0 \\
\hline & De 4.501 a 9.500 & 0 & 0 & 0 & 0 & 0 & 0 \\
\hline & Mais de 9.500 & 0 & 0 & 0 & 0 & 0 & 0 \\
\hline & Não sabe/não respondeu & 4 & 12,5 & 6 & 33,3 & 10 & 20,0 \\
\hline \multirow{4}{*}{$\begin{array}{l}\text { Escolaridade em anos } \\
\text { de aproveitamento } \\
\qquad(p=0,031)^{*}\end{array}$} & Até 4 anos & 24 & 75,0 & 7 & 38,9 & 31 & 62,0 \\
\hline & & & & & & & \\
\hline & Mais de 4 anos & 3 & 9,4 & 6 & 33,3 & 9 & 18,0 \\
\hline & Não sabe/Não respondeu & 5 & 15,6 & 5 & 27,8 & 10 & 20,0 \\
\hline
\end{tabular}

* Teste do Qui-Quadrado $(\alpha=0,05)$ 
Tabela 3. Relação entre o uso clínico de prótese dentária e a autopercepção em saúde bucal dos idosos. Marajó, Pará, 2011-2013

\begin{tabular}{|c|c|c|c|c|c|c|c|}
\hline \multirow{2}{*}{\multicolumn{2}{|c|}{ Autopercepção }} & \multicolumn{6}{|c|}{ Uso de Prótese Dentária } \\
\hline & & \multicolumn{2}{|c|}{$\begin{array}{c}\text { Não usa em nenhuma das } \\
\text { arcadas }\end{array}$} & \multicolumn{2}{|c|}{$\begin{array}{l}\text { Usa em pelo menos } \\
\text { uma das arcadas }\end{array}$} & \multicolumn{2}{|c|}{ Total } \\
\hline \multirow{4}{*}{$\begin{array}{c}\text { Considera que necessita de } \\
\text { tratamento dentário atualmente } \\
(\mathrm{p}=0,534)^{*}\end{array}$} & & (n) & $(\%)$ & (n) & $(\%)$ & (n) & $(\%)$ \\
\hline & Não & 7 & 21,9 & 6 & 33,3 & 13 & 26,0 \\
\hline & Sim & 24 & 75,0 & 12 & 66,7 & 36 & 72,0 \\
\hline & Não sabe/Não respondeu & 1 & 3,1 & 0 & 0 & 1 & 2,0 \\
\hline \multirow{3}{*}{$\begin{array}{c}\text { Considera que necessita usar } \\
\text { prótese total ou trocar a } \\
\text { atualmente usada } \\
(p=0,003)^{*}\end{array}$} & Não & 17 & 53,1 & 2 & 11,1 & 19 & 38,0 \\
\hline & Sim & 15 & 46,9 & 16 & 88,9 & 31 & 62,0 \\
\hline & Não sabe/não respondeu & 0 & 0 & 0 & 0 & 0 & 0 \\
\hline \multirow{3}{*}{$\begin{array}{l}\text { Teve dificuldade de falar por causa } \\
\text { dos dentes } \\
(\mathrm{p}=0,015)^{*}\end{array}$} & Não & 10 & 31,2 & 12 & 66,7 & 22 & 44,0 \\
\hline & Sim & 22 & 68,8 & 6 & 33,3 & 28 & 56,0 \\
\hline & Não sabe/não respondeu & 0 & 0 & 0 & 0 & 0 & 0 \\
\hline \multirow{3}{*}{$\begin{array}{l}\text { Os dentes já fizeram sentir } \\
\text { vergonha de falar ou sorrir } \\
\qquad(p=0,869)^{*}\end{array}$} & Não & 17 & 53,1 & 10 & 55,6 & 27 & 54,0 \\
\hline & Sim & 15 & 46,9 & 8 & 44,4 & 23 & 46,0 \\
\hline & Não sabe/não respondeu & 0 & 0 & 0 & 0 & 0 & 0 \\
\hline \multirow{3}{*}{$\begin{array}{c}\text { Os dentes atrapalham para } \\
\text { trabalhar, estudar ou fazer tarefas } \\
\text { do trabalho/escola } \\
(\mathrm{p}=0,036)^{*}\end{array}$} & Não & 17 & 53,1 & 16 & 88,9 & 33 & 66,0 \\
\hline & Sim & 14 & 43,8 & 2 & 11,1 & 16 & 32,0 \\
\hline & Não sabe/não respondeu & 1 & 3,1 & 0 & 0 & 1 & 2,0 \\
\hline
\end{tabular}

* Teste do Qui-Quadrado $(\alpha=0,05)$ 
Tabela 4. Relação entre a necessidade de prótese dentária e fatores socioeconômicos dos idosos. Marajó, Pará, 2011-2013.

\begin{tabular}{|c|c|c|c|c|c|c|c|}
\hline & & \multicolumn{6}{|c|}{ Necessidade de Prótese Dentária } \\
\hline & & \multicolumn{2}{|c|}{ Não necessita } & \multicolumn{2}{|c|}{$\begin{array}{l}\text { Necessita de PPR } \\
\text { em pelo menos } \\
\text { uma das arcadas }\end{array}$} & \multicolumn{2}{|c|}{$\begin{array}{l}\text { Necessita de } \\
\text { PT em pelo } \\
\text { menos uma } \\
\text { das arcadas }\end{array}$} \\
\hline & & (n) & $(\%)$ & (n) & $(\%)$ & (n) & $(\%)$ \\
\hline \multirow{8}{*}{$\begin{array}{l}\text { Renda familiar total em reais } \\
\text { do mês anterior } \\
(\mathrm{p}=0,304)^{*}\end{array}$} & Até 250 & 0 & 0 & 2 & 7,4 & 0 & 0 \\
\hline & De 251 a 500 & 0 & 0 & 4 & 14,8 & 5 & 22,7 \\
\hline & De 501 a 1.500 & 0 & 0 & 17 & 63,0 & 9 & 40,9 \\
\hline & De 1.501 a 2.500 & 0 & 0 & 1 & 3,7 & 2 & 9,1 \\
\hline & De 2.501 a 4.500 & 0 & 0 & 0 & 0 & 0 & 0 \\
\hline & De 4.501 a 9.500 & 0 & 0 & 0 & 0 & 0 & 0 \\
\hline & Mais de 9.500 & 0 & 0 & 0 & 0 & 0 & 0 \\
\hline & Não sabe/não respondeu & 1 & 100 & 3 & 11,1 & 6 & 27,3 \\
\hline \multirow{3}{*}{$\begin{array}{l}\text { Escolaridade em anos de } \\
\text { aproveitamento } \\
(p=0,125)^{*}\end{array}$} & Até 4 anos & 0 & 0 & 20 & 74,1 & 11 & 50,0 \\
\hline & Mais de 4 anos & 0 & 0 & 4 & 14,8 & 5 & 22,7 \\
\hline & Não sabe/Não respondeu & 1 & 100 & 3 & 11,1 & 6 & 27,3 \\
\hline
\end{tabular}

* Teste do Qui-Quadrado $(\alpha=0,05) \quad$ PPR: Prótese Parcial Removível; PT: Prótese Total. 
Tabela 5. Relação entre a necessidade de prótese dentária e a autopercepção em saúde bucal dos idosos. Marajó, Pará, $2011-2013$.

\begin{tabular}{|c|c|c|c|c|c|c|c|}
\hline \multirow{3}{*}{\multicolumn{2}{|c|}{ Autopercepção }} & \multicolumn{6}{|c|}{ Necessidade de Prótese Dentária } \\
\hline & & \multicolumn{2}{|c|}{$\begin{array}{l}\text { Não necessita de } \\
\text { prótese dentária }\end{array}$} & \multicolumn{2}{|c|}{ Necessita de PPR } & \multicolumn{2}{|c|}{$\begin{array}{c}\text { Necessita de } \\
\text { PT }\end{array}$} \\
\hline & & (n) & $(\%)$ & (n) & $(\%)$ & $(\mathbf{n})$ & $(\%)$ \\
\hline $\begin{array}{c}\text { Considera que necessita de } \\
\text { tratamento dentário } \\
\text { atualmente } \\
(\mathrm{p}=0,169)^{*} \\
\end{array}$ & $\begin{array}{c}\text { Não } \\
\text { Sim } \\
\text { Não sabe/Não respondeu } \\
\end{array}$ & $\begin{array}{l}1 \\
0 \\
0\end{array}$ & $\begin{array}{c}100,00 \\
0 \\
0 \\
\end{array}$ & $\begin{array}{c}4 \\
22 \\
1 \\
\end{array}$ & $\begin{array}{c}14,8 \\
81,5 \\
3,7 \\
\end{array}$ & $\begin{array}{c}8 \\
14 \\
0 \\
\end{array}$ & $\begin{array}{c}36,4 \\
63,6 \\
0 \\
\end{array}$ \\
\hline $\begin{array}{l}\text { Considera que necessita usar } \\
\text { prótese total ou trocar a } \\
\text { atualmente usada } \\
(p=0,354)^{*}\end{array}$ & $\begin{array}{c}\text { Não } \\
\text { Sim } \\
\text { Não sabe/não respondeu }\end{array}$ & $\begin{array}{l}1 \\
0 \\
0\end{array}$ & $\begin{array}{c}100 \\
0 \\
0\end{array}$ & $\begin{array}{c}11 \\
16 \\
0\end{array}$ & $\begin{array}{c}40,7 \\
59,3 \\
0\end{array}$ & $\begin{array}{c}7 \\
15 \\
0\end{array}$ & $\begin{array}{c}31,8 \\
68,2 \\
0\end{array}$ \\
\hline $\begin{array}{l}\text { Teve dificuldade de falar por } \\
\text { causa dos dentes } \\
(\mathrm{p}=0,016)^{*}\end{array}$ & $\begin{array}{c}\text { Não } \\
\text { Sim } \\
\text { Não sabe/não respondeu } \\
\end{array}$ & $\begin{array}{l}1 \\
0 \\
0\end{array}$ & $\begin{array}{c}100,0 \\
0 \\
0 \\
\end{array}$ & $\begin{array}{c}7 \\
20 \\
0\end{array}$ & $\begin{array}{c}25,9 \\
74,1 \\
0 \\
\end{array}$ & $\begin{array}{c}14 \\
8 \\
0\end{array}$ & $\begin{array}{c}63,6 \\
36,4 \\
0 \\
\end{array}$ \\
\hline $\begin{array}{l}\text { Os dentes já fizeram sentir } \\
\text { vergonha de falar ou sorrir } \\
\qquad(\mathrm{p}=0,636) *\end{array}$ & $\begin{array}{c}\text { Não } \\
\text { Sim } \\
\text { Não sabe/não respondeu } \\
\end{array}$ & $\begin{array}{l}1 \\
0 \\
0\end{array}$ & $\begin{array}{c}100,0 \\
0 \\
0 \\
\end{array}$ & $\begin{array}{c}14 \\
13 \\
0\end{array}$ & $\begin{array}{c}51,9 \\
48,1 \\
0 \\
\end{array}$ & $\begin{array}{c}12 \\
10 \\
0\end{array}$ & $\begin{array}{c}54,5 \\
45,5 \\
0\end{array}$ \\
\hline $\begin{array}{l}\text { Os dentes atrapalham para } \\
\text { trabalhar, estudar ou fazer } \\
\text { tarefas do trabalho/escola } \\
\qquad(\mathrm{p}=0,094)^{*}\end{array}$ & $\begin{array}{c}\text { Não } \\
\text { Sim } \\
\text { Não sabe/não respondeu }\end{array}$ & $\begin{array}{l}1 \\
0 \\
0\end{array}$ & $\begin{array}{c}100,0 \\
0 \\
0\end{array}$ & $\begin{array}{c}14 \\
13 \\
0\end{array}$ & $\begin{array}{c}51,9 \\
48,1 \\
0\end{array}$ & $\begin{array}{c}18 \\
3 \\
1\end{array}$ & $\begin{array}{c}81,8 \\
13,6 \\
4,5\end{array}$ \\
\hline
\end{tabular}

*Teste do Qui-Quadrado $(\alpha=0,05) \quad$ PPR: Prótese Parcial Removível; PT: Prótese Total. 
RDAPO: Revista Digital da Academia Paraense de Odontologia

Belém-PA, v.2, n.1, jan./jun. 2018 